\title{
Open Problems Wiki ${ }^{\star}$
}

\author{
Marcus Raitner \\ University of Passau, D-94032 Passau, Germany \\ Marcus . Raitner@Uni-Passau.De
}

\begin{abstract}
This project was inspired by the last year's paper on Selected Open Problems in Graph Drawing by Brandenburg et al. (Proc. 11th GD. Vol. 2919 of LNCS. (2003) 515-539). While being a very good start, a paper is inherently static and will become out-dated. For dynamic content, what open problems (hopefully) are, a web-site is more appropriate. Keeping such a site up-to-date, however, is time consuming and requires good knowledge of recent work. In projects like the free encyclopedia Wikipedia these obstacles are overcome with a collaborative approach: everyone is allowed, and even requested, to contribute his knowledge to the site. The Open Problems Wiki makes use of this paradigm to provide a forum for collecting open problems in graph drawing.
\end{abstract}

\section{Introduction}

Recently, collaboratively edited projects on the WWW were impressively successful; by the time of writing, the free encyclopedia Wikipedia, for instance, has more than 340000 articles in the English version (with translations in over 80 languages in steady progress), edited by over 100000 users. The Wikipedia project is a so-called Wiki, a simple form of content management system where everyone not only is allowed to but also is requested to create new pages or edit existing pages. Meaning "super fast", the Hawaiian word "wiki wiki" was used for this kind of software, because the pages are written in a very simple, yet powerful, markup language; the wiki software then stores the source code in a database, from where it is read and transformed to standard HTML.

It seems that a Wiki is an appropriate framework for collecting and discussing open problems in graph drawing. The task of editing is balanced among the whole community; therefore, it tends to be more comprehensive and more upto-date than other solutions. This wiki shall become the primary site for the open problems in graph drawing, a site everyone knows and contributes to.

\section{Features}

The Open Problems Wiki uses the MediaWiki software, which was developed for the Wikipedia project and is by far the most advanced wiki software available. It already has proved its qualities; it is well documented and supported, and

\footnotetext{
* http://problems.graphdrawing.org 
freely available under the terms of the GNU General Public License (GPL). The following is only a small fraction of the features; a more detailed description can be found in the "Help" section of the wiki.

Users. Although it is not required to register or log in - not even for editing pages - there are many reasons to do so. A registered user can pick a username; all edits, made while being logged in, will be assigned to that name, giving the user full credit for each contribution in the page history (when not logged in, the edits are just assigned to the respective IP address). When logged in, all own contributions are accessible via the "My contributions" link. Many features that are only available to registered users: for example, registered users can mark edits as minor. Minor edits can be filtered from the list of "Recent changes". One very important feature, which active contributors will likely use a lot, are watchlists. When logged in a new link "Watch this page" is shown on every page. That link adds a page to the user's watchlist, which thus becomes basically a filtered view of the "Recent changes" page, showing changes recently made to items in the watchlist. Only registered users are allowed to rename pages, a feature that is very important to maintain structure and consistency. Also, images can be uploaded only by registered users.

Basic Markup. As the pages are stored in a database rather than as files on the server, links between the pages are established via the names of the pages. For instance, [Graph Drawing]] in the source code results in a link to a page with title "Graph Drawing"; if this page does not yet exist, the link leads to a new page with a text field for editing. Most pages are written in a simple markup language, which is sufficient for standard editing tasks like headings (== heading ==), highlighting text ('italic', and ', 'bold', '), or even simple tables. Also, standard HTML can be used for more advanced tasks.

Formulas. The main reason for choosing the same software as in the Wikipedia project was its integrated support for $\mathrm{T}_{\mathrm{E}} \mathrm{X}$-formulas. $\mathrm{T}_{\mathrm{E}} \mathrm{X}$-source within special tags $(<$ math $></$ math $>)$ is rendered on the server and included as Portable Network Graphics (PNG) picture.

History. Since everyone is allowed to edit a page, it is very important to have a sophisticated version control system. The software used for the Open Problems Wiki stores the complete editing history of every page and allows to rollback a page to any previous state.

\section{Acknowledgments}

I would like to thank Ulrik Brandes for supporting the project with the problems subdomain of www.graphdrawing.org.

\section{References}

1. Brandenburg, F.J., Eppstein, D., Goodrich, M.T., Kobourov, S., Liotta, G., Mutzel, P.: Selected open problems in graph drawing. In Liotta, G., ed.: Proc. 11th Intl. Symposium on Graph Drawing (GD). Volume 2919 of LNCS. (2003) 515-539 